\title{
Estudio de la valoración del dolor articular y del grado de satisfacción, tras infiltración con plasma rico en fibrina en pacientes con artrosis de rodilla.
}

\author{
DOI: http//dx.doi.org/10.37315/SOTOCAV20202835573 \\ RODRIGO PÉREZ JL ${ }^{1,2}$, GARCÍA ÁlVAREZ $\mathrm{J}^{1}$, PUIG CONCA $\mathrm{A}^{3}$, JORDÁ LLONA $\mathrm{M}^{2,3}$, NOVOA PARRA C ${ }^{4}$. \\ 1. SERVICIO DE CIRUGÍA ORTOPÉDICA Y TRAUMATOLOGÍA. HOSPITAL UNIVERSITARIO DR. PESET. VALENCIA \\ 2. FACULTAD DE MEDICINA Y ODONTOLOGÍA. UNIVERSIDAD DE VALENCIA \\ 3. SERVICIO DE MEDICINA FÍSICA Y REHABILITACIÓN HOSPITAL UNIVERSITARIO DR. PESET. VALENCIA \\ 4. SERVICIO DE CIRUGÍA ORTOPÉDICA Y TRAUMATOLOGÍA. HOSPITAL DE LA MARINA. DENIA. ALICANTE
}

\begin{abstract}
Resumen
La definición de plasma rico en plaquetas sigue siendo controvertida, quizás el concepto más amplio lo podría definir como "un volumen de plasma autólogo que contiene una concentración de plaquetas superior a la concentración basal fisiológica". En nuestro hospital se realizó un estudio retrospectivo observacional analítico para valoración de resultados y satisfacción de los pacientes tras infiltración con Plasmas Rico en Fibrina (PRF) en rodilla. Se seleccionaron 80 pacientes con diagnóstico de gonartrosis, tratados mediante infiltración de PRF entre enero y diciembre de 2017 por los Servicios de Cirugía Ortopédica y Rehabilitación, que acudieron a las visitas sucesivas de control evolutivo tras la infiltración. Se desestimaron los casos en los que no fue posible un adecuado seguimiento clínico. Respecto al dolor antes del tratamiento, valorado mediante la Escala Numérica del Dolor, la puntuación media fue de 8,48 puntos, observándose una disminución estadísticamente significativa $(p<0,001)$ tras el tratamiento en la media hasta 4,87 puntos, siendo la mediana post infiltración de 5,00 puntos. En cuanto al cambio observado en el perímetro de marcha, la mayoría de los pacientes caminaba 30 minutos con una media de 55,3 minutos y tras la infiltración se observó un incremento de la autonomía de marcha duplicándose la mediana a 60 minutos, con una media de 93,15 minutos de marcha sin dolor. Este incremento de 37,8 minutos en la capacidad de caminar supone una mejoría estadísticamente significativa $(p<0,001)$. También se objetivó una disminución significativa $(p<0,001)$ en el consumo de analgésicos por parte del paciente tras el tratamiento pasando de necesitar analgesia el $81,3 \%$ de los pacientes $(n=61)$ a tan solo el $49,3 \%$ ( $n=37$ ). Por lo tanto, existe una mejoría significativa del dolor, el perímetro de marcha y del reporte del uso absoluto de analgésicos, y no hay ninguna variable asociada previa a la infiltración que explique el resultado.
\end{abstract}

Palabras clave: growth factors, fibrin rich plasma, knee osteoarthritis

\section{Summary}

The definition of platelet rich plasma (PRP) is still controversial, though it can be described in general terms as "a volume of autologous plasma that contains a higher platelet concentration than the physiological basal concentration". A retrospective observational analytical study was carried out in our hospital in order to assess the results and satisfaction of patients that had undergone PRF injection in knee. Between January and December of 2017, 80 gonoarthrosis patients treated with PRF injection were selected by the Orthopaedic Surgery and Rehabilitation Department, all of whom attended several medical appointments to check their recovery. Those cases that did not follow an appropriate clinical follow-up were dismissed from the study. Pain before treatment was assessed by the Numeric Rating Scale, with a mean of 8.48. A statistically significant decrease was observed after treatment $(p<0.001)$, with a 4.87 mean and a 5.00 post-injection median. Regarding walking perimeter, most patients walked a median of 30 minutes and 55.3 minute mean, and their walking autonomy increased after treatment, doubling the median to 60 minutes, with an average of 93.15 minutes of painfree walking. This 37.8 minute increase in the walking ability implies a statistically significant improvement $(p<0.001)$. A significant decrease in analgesics intake after treatment was also observed, being $81.3 \%$ patients who needed analgesia prior to treatment $(n=61)$, and just $49.3 \%(n=37)$ after the procedure. Consequently, there is a significative improvement of pain, walking perimeter and absolute intake of analgesics that cannot be explained by any variable prior to PRF injection.

\section{Correspondencia:}

José Luis Rodrigo Pérez 


\section{Introducción}

La artrosis, es una enfermedad muy prevalente en nuestro medio, siendo la enfermedad músculo-esquelética más común, producida por un desequilibrio entre la usura del cartílago y su regeneración. Existen una serie de factores de riesgo, como la obesidad o las secuelas de traumatismos, que pueden desencadenar un inicio temprano de la enfermedad, teniendo cada vez más importancia los cambios y modificaciones a nivel molecular.

Para conocer la prevalencia de la artrosis de rodilla, lo primero que tenemos que concretar, es bajo qué criterios la diagnosticamos, la mayoría de las publicaciones las hacen con un criterio combinado de dolor de rodilla (expresado bajo la escala visual analógica) y los parámetros radiológicos de Kellgren y Lawrens ${ }^{1}$. Tenemos varios criterios que se asumen por la repetición de los mismos en la bibliografía, como que la artrosis de rodilla es una enfermedad que aumenta con la edad, y que afecta sobre todo a personas mayores de 60 años, y más a las mujeres que a los hombres.

El tratamiento definitivo de la gonartrosis, cuando la correlación clínico-radiológica, sumada a la limitación funcional, no se controla con la toma de anti inflamatorios no esteroideos (AINES), es la artroplastia total de rodilla, con unos índices de éxito sobresalientes. Sin embargo, hay una edad intermedia, en la cual el tratamiento quirúrgico mediante una prótesis de rodilla, no es de elección (por la durabilidad de la misma) que podríamos definir entre 40 y 60 años, y que no conocemos exactamente la prevalencia de la misma.

En un trabajo de Comas y cols. ${ }^{2}$, se señalan varios datos sobre prevalencia de la artrosis, en función de los criterios que se utilicen para su diagnóstico, y llama la atención que en los 2 estudios con pacientes de 40 a 65 años, nos encontramos con una prevalencia en hombres y mujeres de 10 y $18 \%$ respectivamente. Justo en ese tramo de edad de 40 a 65 años donde la población es activa y en edad laboral, es donde los cirujanos ortopédicos intentamos no poner soluciones quirúrgicas como la artroplastia de rodilla, utilizando las alternativas terapéuticas más conservadoras, como infiltraciones con corticoides o visco suplementación con ácido hialurónico

De entre todas ellas los plasmas ricos en plaquetas y fibrina están jugando un papel cada vez más importante en su tratamiento, debido a varias razones entre la que destacan aquellas moléculas que favorecen la curación de los tejidos dañados, mediante procesos de diferenciación, proliferación y síntesis de matriz extracelular. El plasma rico en fibrina, obtenido mediante el sistema VIVOSTAT, es un concentrado de plaquetas y fibrina, definido como un medicamento de uso humano cuya elaboración se realiza mediante un sistema cerrado, y que tiene varias aplicaciones en ortopedia, de entre la que destaca la reparación del cartílago articular.

En un artículo publicado por Kazemi y cols. ${ }^{3}$ en 2015 , se evidencia sobre lesiones condrales en modelo animal, la reparación a los 3 meses de dicho cartílago tanto a nivel morfológico como a nivel microscópico, por lo que se deduce que al menos a nivel experimental hay un efecto beneficioso sobre el cartílago

El objetivo del trabajo es valorar el resultado clínico y la satisfacción del paciente diagnosticado de artrosis de rodilla tras 3 infiltraciones de plasma rico en fibrina.

\section{Material y Método}

Se trata de un estudio analítico retrospectivo observacional de valoración de resultados y satisfacción de los pacientes tras infiltración con Plasma Rico en Fibrina en pacientes con artrosis de rodilla, donde a todos ellos se les pregunto expresamente, o entrego un consentimiento informado para garantizar, la protección de datos según la normativa Ley orgánica 3/2018. Al ser un estudio retrospectivo, en donde la parte fundamental era la revisión de Historias Clínicas, sin ninguna fase de intervención sobre el paciente, fuera de la práctica clínica habitual, no fue necesaria su remisión al Comité Ético (CEIC) del hospital.

Así mismo, no existe ningún conflicto de interés de los autores con el producto en estudio, ni ha sido financiado este trabajo por ninguna empresa relacionada con el sector sanitario.

Se seleccionaron todos los pacientes con diagnóstico de gonartrosis grados 2,3,4 de Kellgreen, tratados mediante infiltración de plasma rico en fibrina, entre enero y diciembre de 2017 , revisados durante el año 2019, por el Servicio de Cirugía Ortopédica y Traumatología y el Servicio de Medicina Física y Rehabilitación del Hospital Universitario Dr. Peset de Valencia y que acudieron a las visitas sucesivas de control evolutivo tras la infiltración. Se desestimaron los casos en los que no fue posible un adecuado seguimiento clínico.

Los criterios utilizados para la selección de pacientes fueron:

\section{Criterios de inclusión:}

-Paciente con diagnóstico de Gonartrosis uní o bilateral

-Edad comprendida entre 30 Y 75 años

-Aceptación de participación en el estudio

-Mantener capacidad cognitiva para responder adecuadamente a la encuesta

\section{Criterios de exclusión:}

-Tratamiento posterior a la infiltración que impida la valoración actual del proceso

-Deterioro cognitivo que invalide los cuestionarios administrados

-Falta de colaboración del paciente

Se sometió a los pacientes a infiltración de plasma rico en fibrina (PRF) en quirófano, realizando una extracción de 120 centímetros cúbicos de sangre periférica, y se procesó 
en el sistema PRF "VIVOSTAT", obteniéndose después de 25 minutos $7 \mathrm{cc}$ de plasma rico en fibrina.

Posteriormente se realizó una infiltración utilizando la vía antero externa sobre el fondo de saco de la articulación de la rodilla (Fig. 1). Esta maniobra se repitió en 2 ocasiones más, espaciadas entre 4 y 6 semanas.

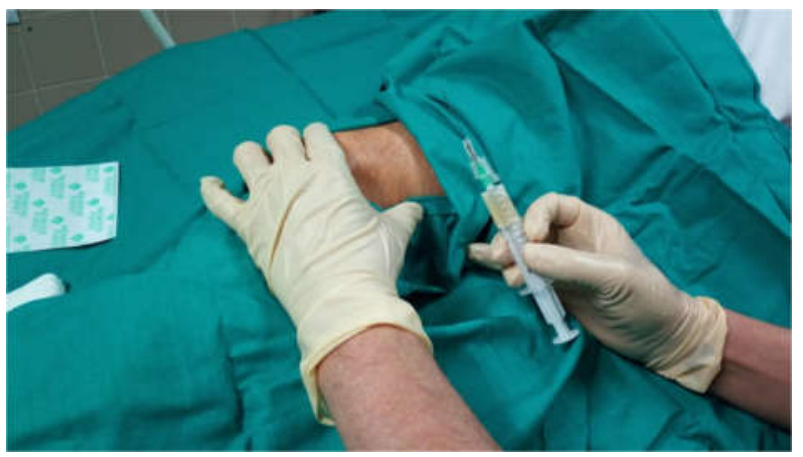

Figura 1: Vía antero-externa para infiltración de la rodilla

La recogida de datos se realizó mediante visita médica para valoración de las siguientes variables:

Edad, sexo, peso, grado de artrosis, intensidad y tiempo de evolución del dolor, perímetro de marcha y tratamiento farmacológico analgésico, todo ello pre y post infiltración y una encuesta de satisfacción con el tratamiento recibido.

El grado de artrosis se determinó mediante la Clasificación Radiológica de la Artrosis de Kellgren y Laurence que establece grados de 0 a 4 según criterios radiológicos.

El dolor fue cuantificado mediante Escala Numérica del Dolor: Escala numerada del 1-10, donde 0 es la ausencia y 10 la mayor intensidad, seleccionando el paciente el número que mejor evalúa la intensidad del síntoma antes de la infiltración y en el momento actual.

El perímetro de marcha se midió en minutos y el tratamiento farmacológico mediante respuesta de "si" o "no" necesita medicación analgésica habitual.

La encuesta de satisfacción fue de elaboración propia mediante respuesta a las preguntas que aparecen en la TABLA 1.

\begin{tabular}{|l|l|l|}
\hline $\begin{array}{l}\text { ¿ESTA SATISFECHOCONEL } \\
\text { RESULTADODELA } \\
\text { INFILTRACION? }\end{array}$ & SI & NO \\
\hline $\begin{array}{l}\text { CESPERABA MEJORES } \\
\text { RESULTADOS? }\end{array}$ & $\mathrm{SI}$ & NO \\
\hline $\begin{array}{l}\text { ¿SE VOLVERIAATRATARCON } \\
\text { INFILTRACION DE PRF? }\end{array}$ & $\mathrm{SI}$ & NO \\
\hline $\begin{array}{l}\text { CRECOMENDARIAEL } \\
\text { TRATAMIENTO AUN } \\
\text { FAMILIARO AMIGO, CONUN } \\
\text { PROBLEMA SIMILAR? }\end{array}$ & SI & NO \\
\hline
\end{tabular}

Tabla I: Encuesta de satisfacción.
El análisis estadístico se llevó a cabo mediante los programas SPSS v.22 y MedCalc v.13. La normalidad de las variables se testó con la prueba de KolmogorovSmirnov. En los análisis antes-después, para evaluar las variables continuas se utilizó el test Wilcoxon y para las variables dicotómicas se utilizó el test de McNemar.

Para evaluar la asociación del resultado de los cuestionarios de satisfacción con las características de la muestra se utilizó prueba exacta de Fischer para las variables dicotómicas, la $U$ de Mann-Whitney para las variables continuas y la razón de verosimilitud para las variables categóricas.

Se realizó un análisis basado en las características operativas del receptor (ROC) para las variables edad y grado de artrosis (Kellgreen) en busca de determinar qué punto de corte tenia mejor relación de sensibilidad y especificidad para predecir la insatisfacción.

En todas las pruebas se consideró un nivel de significación p menor de 0,05 bilateral.

\section{Resultados}

Características de la muestra:

De los 80 pacientes seleccionados se excluyeron 5. Uno por enfermedad de Alzheimer, uno por tratarse de una rotura meniscal, 1 porque no llegó a infiltrarse por mejoría y 2 por no colaborar con la encuesta o no acudir a la visita, resultando una muestra final de 75 pacientes.

De estos 75 pacientes, $44(58,7 \%)$ mujeres y $31(41,3 \%)$ hombres. La edad media fue de $61,4 \pm 10$ años. El peso medio fue de $75,3 \pm 10,6 \mathrm{Kg}$.

Todos los pacientes de la muestra presentaron algún grado de artrosis (Kellgreen Lawerns), siendo grado I en 10 $(13.3 \%)$ de los pacientes, grado II en $29(38,7 \%)$, grado III en $26(34,7 \%)$ y grado IV en $10(13,3 \%)$.

Resultados clínicos y de satisfacción:

El dolor en la rodilla valorado a través de la escala EVA pasó de $8,4 \pm 1,4$ (rango $0-10$ ) puntos a $4,8 \pm 3,1$ (0-10) puntos, $p<0,001$.

El perímetro de marcha medido en minutos paso de $55,3 \pm 60,4$ (rango 1-300) a 93,1 $\pm 83,7(1-300), p<0,001$.

Previo a la infiltración $61(81,3 \%)$ de los pacientes tomaban medicación analgésica horaria, posterior a la infiltración solo $37(49,3 \%)$ pacientes tomaban medicación analgésica horaria, $p<0,001$.

Ante la preguntas ¿Está satisfecho con el resultado tras la infiltración de PRP en su rodilla? $54(72 \%)$ de los pacientes respondió afirmativamente. ¿Se volvería a tratar con infiltraciones de PRP si tuviera el mismo problema? 68 $(90,7 \%)$ de los pacientes respondió afirmativamente. ¿Recomendaría el tratamiento con PRP a un amigo o 
RODRIGO PÉREZ JL y COLS. Estudio de la valoración del dolor articular y del grado de satisfacción, tras infiltración con plasma rico en fibrina en pacientes con artrosis de rodilla.

familiar que tuviese el mismo problema? $65(86,7 \%)$ de los pacientes respondió afirmativamente. A pesar de estos resultados satisfactorios, $43(57,3 \%)$ de los pacientes esperaba un mejor resultado que el obtenido.

Variables relacionadas con la insatisfacción de los pacientes:

No encontramos asociación estadística entre la edad del paciente $(p=0,192)$, sexo $(p=0,798)$, peso $(p=0,671)$, grado de artrosis $(p=0,739)$, toma previa de medicación horaria $(p=0,999)$, EVA previo $(p=0,783)$, perímetro de marcha previo $(p=0,995)$ y el resultado de satisfacción medido a través de la pregunta ¿Está satisfecho con el resultado tras la infiltración de PRP en su rodilla?

Puntos de corte de las variables basales edad y grado de artrosis:

Ante la pregunta ¿Qué edad está relacionada con la insatisfacción de los pacientes con algún grado de artrosis radiológica tras la infiltración de $\mathrm{PRP}$ en la rodilla?, el punto de corte con mejor relación de sensibilidad $(42,9 \%)$ y especificidad (87\%) era la edad mayor a 69 años (Fig. 2). Para este punto de corte existió asociación estadística con la insatisfacción tras el procedimiento, $p=0,010$.

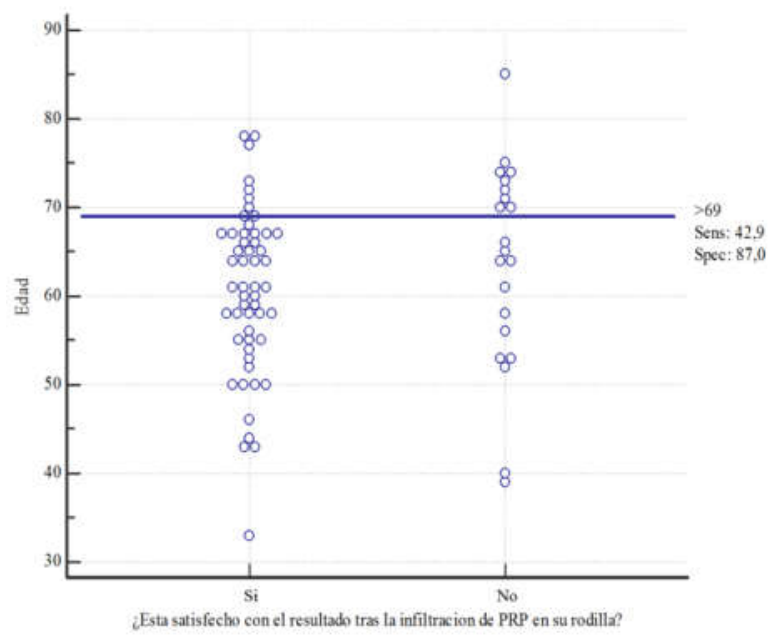

Figura 2: Correlación entre grado de satisfacción con el tratamiento recibido y edad del paciente.

Ante la pregunta ¿Qué grado de artrosis $(\mathrm{KL})$ está relacionada con la insatisfacción de los pacientes tras la infiltración de PRP en la rodilla?, el punto de corte con mejor relación de sensibilidad (55\%) y especificidad $(52,8 \%)$ eran los grados mayores a II de KL (Fig.3). Para este punto de corte no existió asociación estadística con la insatisfacción tras el procedimiento, $p=0,609$.

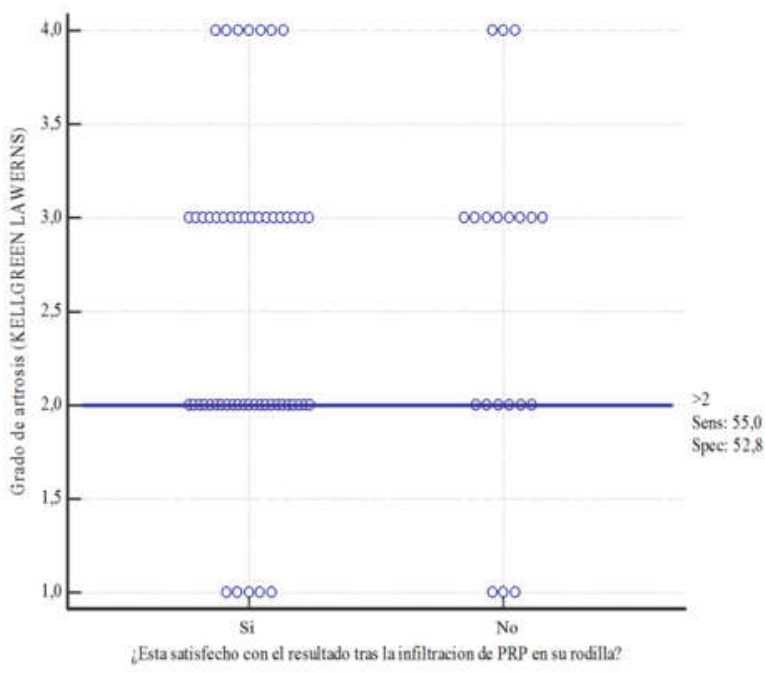

Figura 3: Correlación entre grado de satisfacción y gravedad radiología de la gonartrosis.

\section{Discusión}

La utilización de plasma rico en fibrina y plaquetas están jugando un papel cada vez más importante en el tratamiento de la artrosis, debido a que las plaquetas poseen en sus gránulos una gran cantidad de factores de crecimiento liberados localmente cuando son activadas.

Los más importantes a destacar: factor de crecimiento derivado de las plaquetas ( PDGF), factor de crecimiento transformante beta 1(TGF-1), factor de crecimiento endotelial vascular (VEGF), factor de crecimiento de fibroblastos (FGF),factor de crecimiento epidérmico (EGF), Factor 4, angiopoietinas y endostatinas, además el plasma posee factor de crecimiento de hepatocitos (HGF) y factor de crecimiento insulinoide ( IGF-1), sustancias de interés en la cascada de reparación tisular, son moléculas que estimulan la curación de un tejido dañado, mediante procesos de angiogénesis, miogénesis, quimiotaxis, reclutamiento celular y estímulos para la diferenciación y proliferación celular o síntesis de matriz extracelular.

Hemos encontrado en la literatura médica varios estudios (TABLA II), en los que pacientes con artrosis de rodilla, fueron tratados también con 3 infiltraciones de $\mathrm{PRP} / \mathrm{F}$, con seguimiento y resultados similares a los nuestros.

\begin{tabular}{|l|l|l|l|}
\hline AUTOR & $\begin{array}{l}\mathrm{N}^{\circ} \mathrm{DE} \\
\text { PACINTES }\end{array}$ & $\begin{array}{l}\text { SEGUIMIENTO } \\
\text { MEDIO }\end{array}$ & $\begin{array}{l}\text { \% DE MEJORIA } \\
\text { DEL DOLOR EN } \\
\text { ESCALA EVA }\end{array}$ \\
\hline Buendia (4) & 30 & $12 \mathrm{~m}$ & $10.5 \%$ \\
\hline Sanchez (5) & 60 & $12 \mathrm{~m}$ & $24 \%$ \\
\hline Filardo (6) & 54 & $12 \mathrm{~m}$ & $29 \%$ \\
\hline Spakova (7) & 120 & $6 \mathrm{~m}$ & $45 \%$ \\
\hline Jang (8) & 261 & $12 \mathrm{~m}$ & $32 \%$ \\
\hline Napolitano (11) & 27 & $6 \mathrm{~m}$ & $20 \%$ \\
\hline Gobbi (12) & 29 & $6 \mathrm{~m}$ & $32 \%$ \\
\hline Nuestro estudio & 75 & $19 \mathrm{~m}$ & $36 \%$ \\
\hline
\end{tabular}

Tabla II: Relación de estudios que concluyen con mejoría del dolor tras 3 infiltraciones de PRP/F. 
Buendia $^{4}$ en su trabajo de tesis doctoral, trató a 30 pacientes, con un seguimiento medio de 12 meses, y una disminución del dolor articular medido con la escala EVA, de 6.15 a 5.03 , mientras que Sánchez y cols. ${ }^{5}$ publicaron resultados en 60 pacientes, con una disminución del EVA de 8 a 5.6 tras un seguimiento medio de 12 meses, Filardo ${ }^{6}$ trató a 54 pacientes con un seguimiento medio de 12 meses y una mejora del dolor de 2.9 puntos de la escala EVA, Spakova ${ }^{7}$ tras un seguimiento de 6 meses, consiguió una diminución del EVA de 4.5 puntos en 120 pacientes, y Jang $^{8}$ publicó resultados en 261 pacientes con un seguimiento medio de 12 meses y una mejoría de $7.4 \mathrm{p}$ a 4.2 escala EVA tras el tratamiento.

Otros autores, con la misma pauta de tratamiento consiguieron mejorías porcentuales o estadísticamente significativas del dolor tanto en la escala analógica, como en los ítems específicos de otras escalas de calidad de vida. Así Kon ${ }^{9}$ publicó mejorías estadísticamente significativas del VAS en 50 pacientes con un seguimiento de 6 meses, lo mismo que Wang-Saegusa ${ }^{10}$ con 254 pacientes a los 6 meses, y Napolitano $^{11}$ y Gobbi $^{12}$, consiguieron en 27 y 39 pacientes respectivamente, disminuciones del 20 y $32 \%$ de la Escala VAS.

Los resultados obtenidos demuestran que la utilización del PRF, en la infiltración intraraticular de la artrosis de rodilla es un tratamiento seguro, no tiene efectos secundarios no deseados, y mejora tanto el dolor articular, principal síntoma de la enfermedad, como el perímetro de marcha, y por lo tanto la calidad de vida de nuestros pacientes.

\section{Limitaciones del estudio}

Es un estudio retrospectivo de valoración de resultados, con ausencia de un grupo control (cuestionable por motivos éticos al ser un estudio de superioridad).

Ha habido pérdida de casos, y la muestra es heterogénea.

\section{Conclusiones:}

Es uno de los pocos trabajos que recogen los resultados de satisfacción de un paciente pre y post tratamiento, aportando así una perspectiva desde el punto de vista de nuestros enfermos.

Respecto a los resultados hubo una reducción estadísticamente significativa del dolor en la escala analógica visual. Tras la infiltración con PRF hay una mejoría significativa del dolor, el perímetro de marcha y disminución del uso absoluto de analgésicos horarios, aunque no hay ninguna variable previa a la infiltración que explique el resultado, solo la edad mayor a 69 años y la artrosis grave parece estar asociada a la insatisfacción de los pacientes.

A ninguno de los pacientes incluidos en el estudio ha sido necesario colocarle una artroplastia total de rodilla, para tratar su gonartrosis, por lo que podemos afirmar que retrasa al menos 2 años la necesidad de utilizar implantes.

De forma similar a otros trabajos publicados, se obtienen mejores resultados en pacientes menores de 65 años y con artrosis no severa. No obstante deben continuar trabajos observacionales de obtención de resultados para estandarizar la técnica, indicaciones y la posología adecuada. 
RODRIGO PÉREZ JL y COLS. Estudio de la valoración del dolor articular y del grado de satisfacción, tras infiltración con plasma rico en fibrina en pacientes con artrosis de rodilla.

\section{Bibliografía}

1. Kellgren JH, Lawrence JS. Rheumatism in miners. II. X-ray study. Br J Ind Med 1952; 9:197-207.

2. Comas M, Sala M, Roman R, Hoffmeister L, Castells $\mathbf{X}$. Variaciones en la estimación de la prevalencia de artrosis de rodilla según los criterios diagnosticos utilizados en los estudios poblacionales. Gac San 2010; 24 (1):28-32.

3. Kazemi D, Fakhrjou A. Leukocyte and Platelet Rich Plasma (L-PRP) Versus Leukocyte and Platelet Rich Fibrin (L-PRF) For Articular Cartilage Repair of the Knee: A Comparative Evaluation in an Animal Model. Iranian Red Crescent Medical Journal 2015; 17(10):e19594.

4. Buendia D. Valoración clínica y mediante técnicas de imagen de la evolución de pacientes con gonoartrosis tratados mediante ácido hialuronico y plasma rico en plaquetas. Tesis Doctoral. Universidad de Murcia. Departamento de anatomía humana y psicobiologia. 2015.

5. Sanchez M, Anitua E, Azofra J, Aguirre JJ, Andia I. Intra-articular injection of an autologous preparation rich in growth factors for the treatment of knee OA: a retrospective cohort study, Clin Exp Rheumatol 2008; 26:910-3.

6. Filardo G,Kon E,Buda R, Timoncini A, Di Martino ., Cenacchi A et al. Platelet-rich plasma intra-articular knee injections for the treatment of degenerative cartilage lesions and osteoarthritis. Knee Surg Sports Traumatol Arthrosc Off J ESSKA 2011; 19:528-35.

7. Spakova T, Rosocha J, Lacko M, Harvanova D, Gharaibeh A. Treatment of knee joint osteoarthritis with autologous platelet-rich plasma in comparison with hyaluronic acid. Am J Phys Med Rehabil/Assoc Acad Physiatrists 2012;91(5);411-7.

8. Jang SJ, Kim JD, Cha S. Platelet-rich plasma (PRP) injections as an effective treatment for early osteoarthritis. Eur J Orthop Surg Traumatol 2012; 23:573-80.

9. Kon E, Mandelbaum B, Buda R, Filardo G, Delcogliano M, Timoncini A et al. Platelet-rich plasma intra-articular injection versus hyaluronic acid viscosupplementation as treatments for cartilage pathology: from early degeneration to osteoarthritis. Arthroscopy 2011; 27:1490-501.

10. Wang-Saegusa A, Cugat R, Ares O, Seijas R, Cusco X, Garcia-Balletbo M. Infiltration of plasma rich in growth factors for osteoarthritis of the knee short-term effects on function and quality of life. Arch Orthop Trauma Surg 2011; 131:311-7.

11. Napolitano M, Matera S, Bossio M, Crescibene A, Costabile E, Almolla J et al. Autologous platelet gel for tissue regeneration in degenerative disorders of the knee. Blood Transfus 2012; 10:72-7.

12. Gobbi A, Karnatzikos G, Mahajan V, Malchira S. Platelet-rich plasma treatment in symptomatic patients with knee osteoarthritis: preliminary results in a group of active patients. Sports Health 2012; 4:162-72. 